\title{
Mineração
}

\section{Plano de recuperação de áreas degradadas versus plano de fechamento de mina: um estudo comparativo}

\author{
Hernani Mota de Lima \\ Professor Adjunto do Departamento de Engenharia de Minas (DEMIN) da Escola de Minas/UFOP \\ E-mail: hernani.lima@ufop.br \\ José Cruz do Carmo Flores \\ Professor Assistente do Departamento de Engenharia de Minas (DEMIN) da Escola de Minas/UFOP \\ E-mail: jcruzflores@demin.ufop.br
}

Flávio Luiz Costa

Mestre em Engenharia Mineral - CPGEM, Depart. de Eng. de Minas (DEMIN)/Escola de Minas/UFOP

E-mail:flavio.costa@rdmbr.com.br

\section{Resumo}

O presente artigo relata os resultados de uma análise de 20 Planos de Recuperação de Áreas Degradadas (PRADs) elaborados para minas localizadas no Quadrilátero Ferrífero (MG), de acordo com o Decreto ${ }^{\circ}$ 97.632, de 10 de abril de 1989. Usualmente, PRAD tem sido compreendido como plano de fechamento de mina. Portanto o objetivo desse estudo foi verificar o conteúdo e o cumprimento desses PRADs à Norma NBR 13030 - "Elaboração e apresentação de projeto de reabilitação de áreas degradadas pela mineração", bem como comparar tais conteúdos com o conteúdo típico de um Plano de Fechamento de Mina, estabelecendo similaridades e diferenças entre os instrumentos objetos do estudo. Esse trabalho sintetiza os resultados dessa análise comparativa e evidencia os riscos (ambientais, sócio-econômicos e culturais) de se considerar um PRAD como um Plano de Fechamento de Mina; ressalta a necessidade de discernir PRAD de Plano de fechamento e chama a atenção para a sobreposição de requerimentos às empresas de mineração.

Palavras-chave: Recuperação de áreas degradadas, fechamento de mina, PRAD.

\begin{abstract}
This paper reports the results of an analysis of 20 Plans for Rehabilitation of Degraded Areas (PRADs) prepared for mines located in the Iron Quadrangle $(M G)$ in accordance with the Decree $n^{\circ}$ 97.632, dated April 10th of 1989. Usually PRAD has been understood as a mine closure plan. Therefore, the objective of this study was to check PRADs contents and compliance with the Norm NBR 13030 - "Elaboration and Presentation of rehabilitation project for impacted areas by mining", as well as, to compare its contents with a mine closure plan, in order to establish similarities and differences between them. This work presents the results of this comparative analysis; highlights the environmental, socio-economic and cultural risks of taking a PRAD as a mine closure plan; shows the necessity of differing PRAD from a mine closure plan and stresses the requirements' overlap for mine companies.
\end{abstract}

Keywords: Mine rehabilitation, closure plan. 


\section{Introdução}

A obrigação fundamental imposta aos titulares de concessões de lavra no Brasil, com relação ao fechamento das minas, é que eles promovam a reabilitação das áreas impactadas pelas atividades da mineração, de acordo com um Plano de Recuperação de Áreas Degradadas (PRAD), previamente elaborado e aprovado pelo órgão governamental competente.

A exigência da apresentação obrigatória do PRAD fundamenta-se no princípio de que as áreas ambientalmente perturbadas pelas atividades de mineração devem ser devolvidas à comunidade ou ao proprietário superficiário nas condições desejáveis e apropriadas ao retorno do uso original do solo ou naquelas necessárias para a implantação de outro uso futuro, desde que escolhido por consenso entre as partes envolvidas e afetadas pela mineração.

$\mathrm{O}$ preceito legal estabelece que o PRAD deve considerar a solução técnica adequada, visualizada pela empresa de mineração, para reabilitar o solo, eventualmente degradado pela atividade de mineração, para uso futuro (IBRAM, 1992; DNPM, 2000). O plano aprovado pode ser revisto ou alterado, com a concordância do órgão ambiental competente para sua aprovação, para incorporar inovações tecnológicas ou outras ações alternativas que se mostrem mais adequadas ao processo de reabilitação, à medida que se desenvolvem as atividades de lavra e beneficiamento.

Com o objetivo de avaliar a qualidade e a adequação dos Planos de Recuperação de Áreas Degradas para o propósito do fechamento das minas, quando do término ou paralisação das operações, foram analisados 20 (vinte) PRADs de minas, cujo ciclo produtivo encontra-se próximo do fim, submetidos pelos titulares / operadores das minas à aprovação da Fundação Estadual do Meio Ambiente do Estado de Minas Gerais - FEAM.

Na primeira etapa do estudo, os PRADs foram comparados entre si, com o objetivo de aferir a consistência dos conteúdos e sua adequação à norma NBR 13030. Na segunda, o objeto da comparação foi o conteúdo dos Planos de Recuperação de Áreas Degradadas com o conteúdo típico de um Plano de Fechamento de Mina, com o objetivo de se estabelecerem similaridades e diferenças entre os dois planos.

Apresenta-se, aqui, a síntese dos principais resultados que o estudo comparativo permitiu extrair, após a análise e avaliação dos elementos selecionados para esse fim.

\section{Análise da qualidade dos PRADs}

Muitas das minas consideradas de grande porte localizadas no Estado de Minas Gerais, atualmente próximas da exaustão das reservas e conseqüente fechamento definitivo, já estavam em operação quando passou a vigorar o Decreto $n^{\circ}$ 97.632, de 10 de abril de 1989 , que criou a obrigatoriedade da apresentação do Plano de Recuperação de Áreas Degradadas pelos empreendimentos que se destinam à exploração de recursos minerais, quando da apresentação do Estudo de Impacto Ambiental (EIA) e do Relatório de Impacto Ambiental (RIMA) ao órgão ambiental competente (Decreto n ${ }^{\circ}$ 97.632/89, Art. $1^{\circ}$ ).

Apesar dos bons resultados ambientais que se constatam em algumas minas objeto deste estudo, no geral, a qualidade de seus PRADs é questionável, e muitas falhas foram detectadas na elaboração desses planos. As principais incluem:

a) A ausência de conhecimento prévio das características dos ecossistemas, bem como das práticas e processos de reabilitação de áreas mineradas disponíveis. Quando de sua promulgação, o Decreto estabeleceu um prazo de 180 dias para que as empresas, em operação, apresentassem os respectivos PRADs. Esse curto prazo impossibilitou um levantamento adequado da base de dados ambientais suficiente para a correta e eficaz elaboração desses planos. b) O Decreto Federal de 1989 não cuidou de detalhar as medidas mitigadoras, especificando apenas que " $A$ recuperação deverá ter por objetivo o retorno do sítio degradado a uma forma de utilização, de acordo com um plano preestabelecido para o uso do solo, visando a obtenção de uma estabilidade do meio ambiente" (Decreto $\mathrm{n}^{\circ}$ 97.632/89, Art. $3^{\circ}$ ). $\mathrm{Na}$ verdade, nem para os novos PRADs há ainda clara responsabilidade institucional, no que diz respeito, por exemplo, à exigência de apresentação de garantias de disponibilidade dos recursos financeiros necessários à implantação de todas as ações contratadas, através dos PRADs.

c) Limitação da abordagem multidisciplinar por parte das empresas de consultoria responsáveis pela elaboração dos PRADs.

d) Em alguns casos, as empresas contratadas para elaboração do PRAD tenderam a minimizar o custo do diagnóstico do impacto ambiental do projeto, através da limitação do seu escopo.

e) Não houve, em 1989, audiência pública para discussão dos PRADs apresentados.

f) Os PRADs apresentados ao órgão ambiental não passaram por nenhum processo de análise e julgamento quanto à sua qualidade técnica e capacidade de solução dos problemas detectados.

\section{Eficácia dos PRADs elaborados}

Muitas das grandes minas encontram-se próximas do estágio de fechamento definitivo, como conseqüência da exaustão das reservas, e espera-se que os seus Planos de Recuperação de Áreas Degradadas possam ser usados como diretrizes para as partes - empresas de mineração e órgãos governamentais estabelecerem um programa consensual para o fechamento das minas. Entretanto esta ainda não tem sido a prática ado- 
tada pelas partes. Entre as razões que explicam essa lacuna comportamental, a principal delas é que, como consequiência da carência de recursos humanos e financeiros que aflige as agências ambientais, encarregadas da análise e aprovação dos PRADs, estes, quando submetidos à apreciação, não são analisados e permanecem arquivados nas agências, pendentes de decisão final. Conforme citado por Brandi (1994),os PRADs guardam uma estreita relação de conteúdos com os EIA-RIMAS e poucos são utilizados pelas empresas como orientação aos trabalhos de campo.

Os Planos de Recuperação de Áreas Degradadas e de Fechamento de Mina não devem ser elaborados em determinado momento e, no momento seguinte, quedarem esquecidos, depositados numa prateleira. O planejamento da recuperação das áreas degradadas, compreendido como um dos elementos que integram o plano de fechamento de mina,é um processo contínuo e dinâmico, que pode atravessar décadas, durante as quais muitas variáveis certamente sofrerão mudanças, incluindo os contextos político e social, os institutos legais e a natureza dos controles específicos das indústrias.

\section{Estudo comparativo dos PRADs}

O estudo comparativo dos Planos de Recuperação de Áreas Degradadas selecionados permitiu verificar que o processo de cópia e colagem foi adotado por muitas empresas de consultoria ou por consultores independentes para a elaboração desses documentos. O emprego desse processo fica evidente quando se comparam os PRADs elaborados para minas, minérios e regiões "consideradas" semelhantes. Verificouse, em tais casos, que tópicos como descrição e avaliação dos impactos e descrição das medidas mitigadoras são muito semelhantes. Essa prática não é apropriada, pois cada mina é única na sua essência e está inserida em ecossistema específico, bem como a geologia, mine- ralogia e outras características do minério diferem em muito.

Facilmente se constata, na análise dos PRADs, que muitos deles apresentam uma abordagem superficial e incompleta das variáveis avaliadas e que não foram estruturados sobre as características reais e específicas do sítio a que se referem. Em outras palavras, foram preparados para cumprir a exigência da lei. Adicionalmente, muitos dos PRADs apresentados pelas empresas não contemplavam a proposição de um Sistema de Gerenciamento Ambiental para o sítio considerado.

\section{Plano de recuperação de áreas degradadas versus plano de fechamento de mina}

Um plano de fechamento de mina deve atender às exigências legais, levando em consideração, ao mesmo tempo, as características ambiental, econômica e social específicas de uma mina e de seu entorno, das operações e de toda a infra-estrutura de apoio que integra o projeto de mineração. Portanto o conteúdo dos planos de fechamento sofre variações, para contemplar características locais específicas de cada projeto. Entretanto é possível estabelecer um conteúdo básico que fundamente a estrutura de todos os planos de fechamento.

Nessa etapa do estudo, comparouse o conteúdo típico, básico, de um plano de fechamento de mina (coluna 1) com os conteúdos dos PRADs (coluna 2), com o objetivo de se estabelecerem as similaridades $(\sqrt{ })$ e as diferenças ou ausências ( $\mathbf{X}$ ) existentes entre os conteúdos desses dois instrumentos. Os resultados desse estudo comparativo são apresentados na Tabela 1.

A comparação dos conteúdos dos PRADs por atividades de mineração selecionados com o conteúdo de um Plano Básico de Fechamento de Mina conduziu às seguintes constatações:

\section{l) Objetivos}

Os objetivos dos PRADs são bastante semelhantes, em termos gerais, àqueles propostos nos planos de fechamento de minas. Ambos os instrumentos colocam como, objetivo amplo, a garantia da segurança e da saúde pública, através da reabilitação das áreas perturbadas pela mineração, de modo a retorná-las às condições desejáveis e necessárias à implantação de um uso pós-mineração previamente eleito e socialmente aceitável. Entretanto os meios propostos, para se alcançarem os objetivos divergem muito entre os instrumentos analisados.

\section{II) Agentes envolvidos}

Há consenso que, em princípio, o envolvimento público nos processos de tomada de decisão, implantação de ações e gerenciamento do processo de recuperação de áreas impactadas pela mineração é importante fator para firmar na comunidade o sincero envolvimento da empresa com as questões que lhe afetam e com a proteção ambiental, assim como para demonstrar sua habilidade para conduzir seus negócios de maneira ambientalmente responsável e melhorar continuamente a qualidade das decisões tomadas, com respeito ao gerenciamento ambiental (EPA, 1995; Hannan, 1998; Albeerts \& Grasmick, 2000).

É fundamental, para o sucesso do processo de fechamento de uma mina, que se identifiquem todos agentes envolvidos - principalmente aqueles considerados elementos-chave para o processo - e todas as partes interessadas; e que a empresa mantenha sempre abertos os canais de diálogo e um relacionamento cordial e franco com todos os atores. Embora alguns PRADs analisados façam referências à comunidade diretamente envolvida com a operação da mina, na maioria deles o processo de identificação dos agentes envolvidos, das partes interessadas e de consulta à comunidade, não foi reportado como ações integrantes do planejamento para o fechamento. 
Tabela 1 - Comparação do conteúdo básico de um plano de fechamento de mina com os conteúdos dos PRADs analisados.

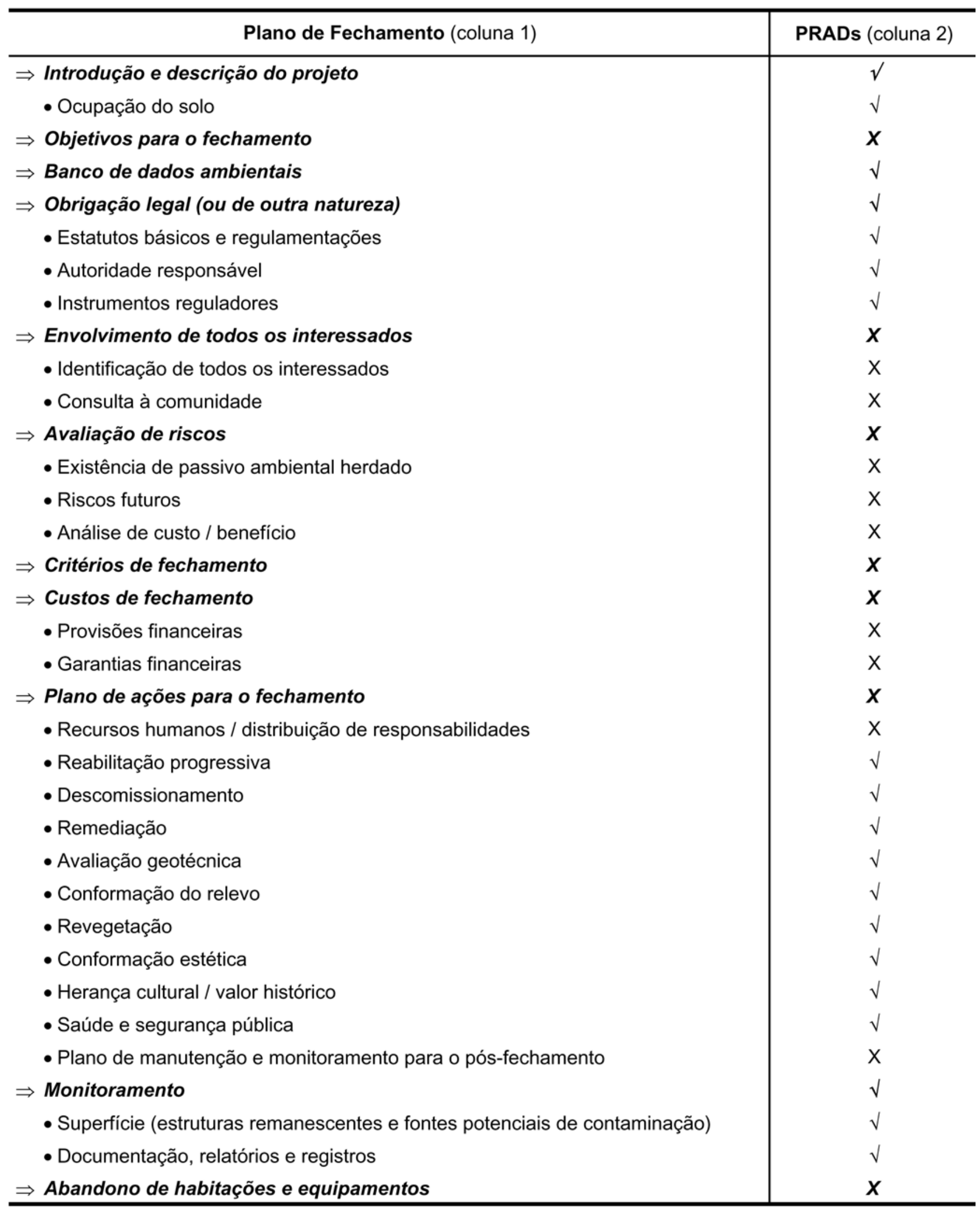

Fonte: Modificada de Lima (2002). 


\section{III) Avaliação de riscos}

A abordagem dos riscos potenciais no fechamento e pós-fechamento da mina, através de um processo de identificação das fontes potenciais, avaliação dos riscos e planejamento das ações mitigadoras adequadas, pode reduzir os custos e as incertezas desses processos.

As modernas tendências identificadas no planejamento do fechamento de minas passam pela revisão técnica e análises de riscos e de custo / benefício, tanto no âmbito técnico, quanto em termos sociais e ambientais. A aplicação da análise de riscos, no planejamento do fechamento das minas, fundamenta-se na quantificação de fatores subjetivos e na análise das incertezas, relacionadas tanto ao desempenho quanto ao custo das ações planejadas e implantadas (Morrey \& van Zil, 1994; Morrey, van Zil et al., 1995; Morrey, 1999).

Nenhum dos PRADs analisados apresenta abordagem do processo de recuperação das áreas degradadas sob a ótica da análise de riscos.

\section{IV) Custos de fechamento}

O objetivo da provisão de recursos financeiros e apresentação de garantias para o propósito específico do fechamento de uma mina é assegurar aos agentes e partes envolvidas que os custos de implantação e gerenciamento do plano de fechamento estão adequadamente contemplados no planejamento financeiro da empresa de mineração e não serão transferidos aos órgãos governamentais ou à comunidade, por ocasião da exaustão das reservas minerais da mina, ou em caso de ocorrer interrupção abrupta da produção, como conseqüência, por exemplo, da falência da empresa de mineração.

Muitos dos PRADs analisados não incluíam a estimativa dos custos de reabilitação com cronograma físico e financeiro e tampouco demonstravam a constituição de fundo ou qualquer outra forma de provisão financeira. Portanto há sempre o risco de que a responsabilida- de pela limpeza e reabilitação do sítio recaia sobre o governo ou a comunidade, no futuro, após o encerramento da fase produtiva do empreendimento.

\section{V) Ações planejadas para o fechamento}

A maioria dos PRADs analisados concebe a reabilitação da mina apenas como um processo de revegetação. Em poucos, entretanto, estão incluídos tópicos como responsabilidades sociais, reabilitação progressiva, descomissionamento, avaliação geotécnica e reconstituição da superfície topográfica, nos seus planos de ações. Em adição, a maioria dos PRADs não apresenta um plano de monitoramento e manutenção das áreas recuperadas, após o fechamento da mina. Item também citado por Brandi (1994) como um dos pontos fracos dos PRADs.

\section{VI) Critério de fechamento e abandono da área}

Devido à ausência da adoção de critérios para a avaliação do sucesso (ou insucesso) do processo de fechamento de mina no Brasil, a maioria dos PRADs não especifica ou menciona os indicadores que serão adotados para demonstrar a conclusão, com sucesso, do processo de reabilitação das áreas ambientalmente alteradas durante a operação da mina. A ausência desses critérios e de uma metodologia para especificá-los é observada, tanto na esfera governamental, quanto no âmbito das empresas de mineração.

O objetivo final de um plano de fechamento de mina é alcançar um ponto onde a companhia demonstre ter concluído, com comprovado sucesso, todas as metas (legais, sociais, ambientais e técnicas) acordadas com as autoridades competentes e comunidades envolvidas, de acordo com um conjunto de critérios previamente enumerados e adotados para essa avaliação.

A expectativa é que os órgãos governamentais aceitem o desempenho das empresas como satisfatório, liberandoas das responsabilidades por elas assumidas. Conseqüentemente, é de se supor que a responsabilidade pela neutralização de qualquer dano ambiental potencial que venha a eclodir na área, no futuro, e os custos da implantação das medidas mitigadoras necessárias, recairão nas contas do Estado ou sobre o proprietário superficiário subseqüente. Em adição, as companhias de mineração acreditam que, amparados por leis ambientais e civis tolerantes, se eximirão de responsabilidades por danos futuros, mas cujas origens encontram-se nas ações ou omissões por elas praticadas no passado (Williams, 1993).

Como o Brasil não adota nenhuma diretriz para o estabelecer critérios de avaliação dos trabalhos implantados e subseqüente liberação das áreas mineradas pelas empresas de mineração e recebimento dessas áreas recuperadas pelas comunidades ou pelos proprietários do solo, não há menção, nos PRADs analisados, aos procedimentos que serão adotados no momento de se concretizar a transferência de responsabilidades.

\section{Conclusão}

Nos países com larga tradição em mineração, um Estudo de Viabilidade Técnica e Econômica completo, necessariamente deve contemplar o Plano de Lavra, o EIA/RIMA, assim como um Plano de Fechamento de Mina, que incluirá, entre outros elementos, o Plano de Descomissionamento e o PRAD. A apresentação desses planos é pré-requisito imposto ao proponente, como condição para a obtenção da concessão de lavra. A obrigatoriedade da apresentação do plano de fechamento constitui, de fato, um dos instrumentos mais comuns de que dispõem os governos para garantir o fechamento completo e seguro das minas.

No Brasil, o PRAD é parte do EIA e, por isso, alguns o entendem como um Plano Conceitual de Fechamento de Mina. Entretanto ressalta-se o quão dis- 
tantes estão os PRADs do conteúdo básico que um Plano de Fechamento de Mina deve apresentar. Esse estudo não visa a tecer críticas sobre os PRADs, embora tenha constatado várias falhas neles. O objetivo principal foi mostrar que planos de fechamento e PRADs são instrumentos distintos e complementares. PRAD é, e assim deve ser considerado, um componente do Plano de Fechamento.

Esse estudo busca chamar atenção para aquilo que, no entender dos autores, constitui uma sobrecarga burocrática: exigir a inclusão de PRADs nos EIAs. Os autores defendem, sim, a exigência do plano de fechamento a ser apresentado na fase de licenciamento do empreendimento. O EIA forma a base de dados para a elaboração de um plano conceitual de fechamento de mina, o qual deverá ser atualizado, periodicamente, de modo a atender às mudanças (sejam elas tecnológicas, de tipo de minério ou de caráter legal) que a mina irá passar ao longo de sua vida útil.

O presente estudo não teve a intenção de fazer uma análise quantitativa dos PRADs. Daí usarem-se, no texto, expressões qualitativas como, por exemplo, "na maioria". O motivo para tal foi, apenas, mostrar as diferenças conceitu- ais e práticas do PRAD para com um plano de fechamento, bem como as lacunas, deficiências e falhas que se pode cometer ao adotar o PRAD como um plano de fechamento de mina.

\section{Referências bibliográficas}

ALBERTS, D., GRASMICK, M. K. Community involvement at Nicolet Minerals. Mining Environmental Management, v. 8, n. 2, p.19-20, 2000.

BRANDI, I. V. Estudo da efetividade dos planos de recuperação de áreas degradadas (PRADs) de atividade de explotação de minério de ferro na região do quadrilátero ferrífero - MG. Escola de Engenharia de São Carlos (USP): 1994. 114p. (Dissertação de Mestrado).

DNPM- Departamento Nacional de Produção Mineral. Investidor guide's. Mining in Brazil. Basic information for the investidor. Information captured at http://www.dnpm.gov.br/. 2000.

BRASIL. Decreto n. 97.632 - 10 abr. 1989. Dispõe sobre a regulamentação do Artigo $2^{\circ}$, inciso VIII, da Lei n. 6.938, de 31 de agosto de 1981, e dá outras providências.

EPA. Community consultation and involvement. The Australian Environment Protection Agency, 1995.

HANNAN, J. C. Community consultation issues in relation to mine closure. Environmental Issues in the
Decommissioning of Mine Sites, Brisbane, Australian Centre for Mining Environmental Research, 1998.

IBRAM. Mineração e meio ambiente. Brasilia: Instituto Brasileiro de Mineração (IBRAM), 1992.

LIMA, H. M. Liability assessment: a tool for mine closure planning. EIA - Unit, Biological Science Department. Aberystwyth: University of Wales Aberystwyth, 2002. p. 197.

MORREY, D. R. Integrated planning for economic environmental management during mining operations and mine closure. Environmental policy in mining: corporate strategy and planning for mining closure. In: WARHURST. A., NORONHA, L. (Ed.). New York: Lewis Publishers. 1999. p. 243-256.

MORREY, D. R., D. van ZIL. Including uncertainty in mine closure. 5th Western Regional Conference on Precious Metals, Coal and the Environment, Black Hills, South Dakota, Society for Mining, Metallurgy and Exploration, 1994.

MORREY, D. R., D. van ZIL, et al. Principal components of economic mine closure. In: CONFERENCE ON MINING AND ENVIRONMENT IN ZIMBABWE, 1. p.184-194, 1995.

WILLIAMS, G. Closing a hardrock mine Can you ever walk away? Rocky Mountain Mineral Law Institutes, 40, 1993.

Artigo recebido em 09/03/2006 e aprovado em 20/09/2006.

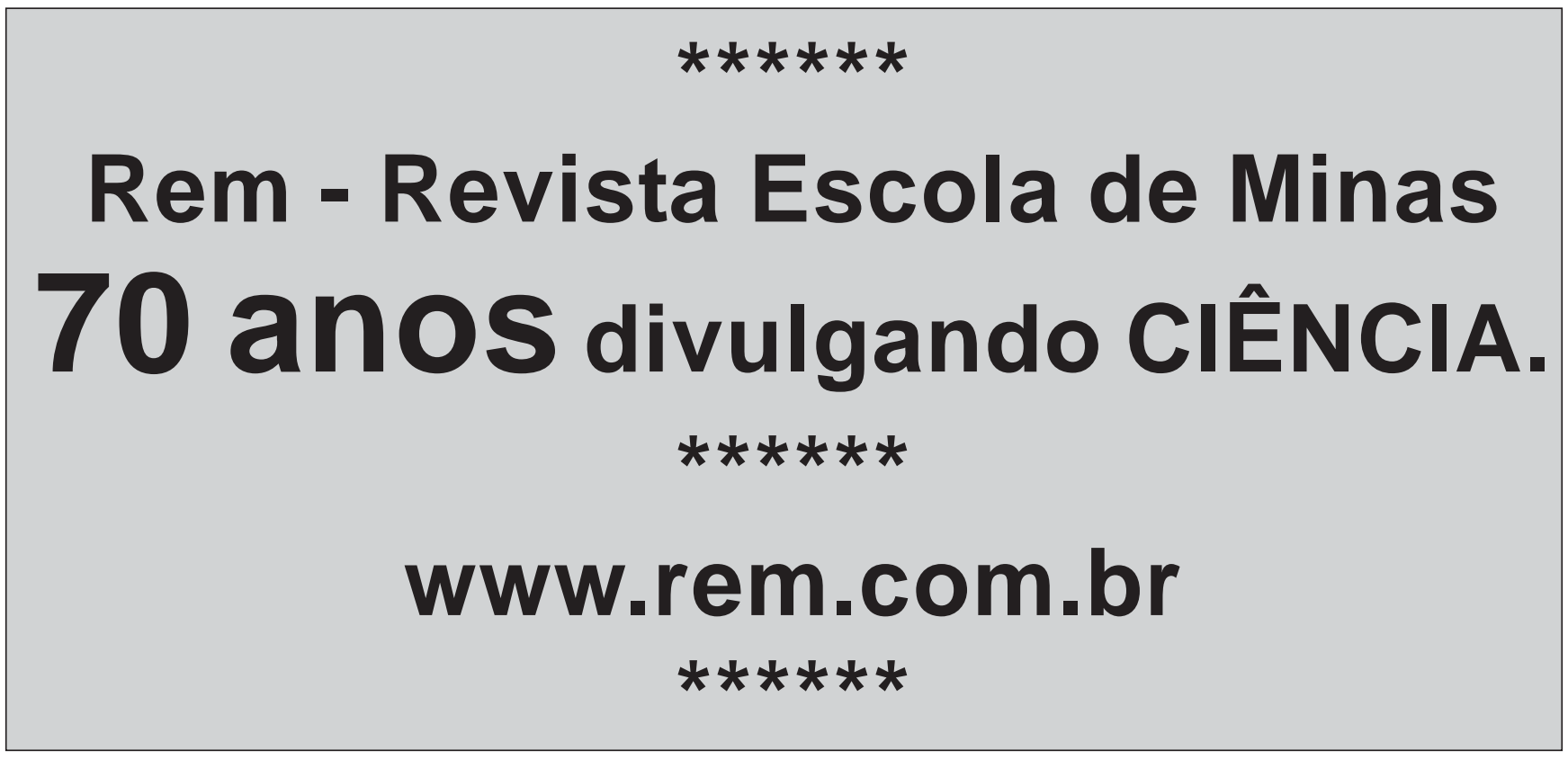

